\title{
Porcine to Human Heart Transplantation: Is Clinical Application Now Appropriate?
}

\author{
Christopher G. A. McGregor ${ }^{1,2}$ and Guerard W. Byrne ${ }^{1,2}$ \\ ${ }^{1}$ Institute of Cardiovascular Science, University College London, London, UK \\ ${ }^{2}$ Department of Surgery, Mayo Clinic, Rochester, MN, USA \\ Correspondence should be addressed to Christopher G. A. McGregor; c.mcgregor@ucl.ac.uk
}

Received 6 July 2017; Accepted 8 October 2017; Published 7 November 2017

Academic Editor: Laura Iop

Copyright ( 2017 Christopher G. A. McGregor and Guerard W. Byrne. This is an open access article distributed under the Creative Commons Attribution License, which permits unrestricted use, distribution, and reproduction in any medium, provided the original work is properly cited.

\begin{abstract}
Cardiac xenotransplantation (CXTx) is a promising solution to the chronic shortage of donor hearts. Recent advancements in immune suppression have greatly improved the survival of heterotopic CXTx, now extended beyond 2 years, and life-supporting kidney XTx. Advances in donor genetic modification (B4GALNT2 and CMAH mutations) with proven Gal-deficient donors expressing human complement regulatory protein(s) have also accelerated, reducing donor pig organ antigenicity. These advances can now be combined and tested in life-supporting orthotopic preclinical studies in nonhuman primates and immunologically appropriate models confirming their efficacy and safety for a clinical CXTx program. Preclinical studies should also allow for organ rejection to develop xenospecific assays and therapies to reverse rejection. The complexity of future clinical CXTx presents a substantial and unique set of regulatory challenges which must be addressed to avoid delay; however, dependent on these prospective life-supporting preclinical studies in NHPs, it appears that the scientific path forward is well defined and the era of clinical CXTx is approaching.
\end{abstract}

\section{Introduction}

About 5.7 million Americans have heart failure, half of whom will die within 5 years [1]. Organ transplantation is currently the preferred solution for treatment of end-stage heart failure but less than 3000 heart transplants have been performed annually in the US in recent years. Circulatory assist devices and total artificial hearts have been approved to support patients in chronic heart failure $[2,3]$. These mechanical solutions are effective, at least in the short term, but have significant morbidity from thromboembolism, infection, gastrointestinal bleeding, and reduced quality of life [4]. Regenerative solutions for heart failure remain a nascent experimental technology. Cardiac xenotransplantation (CXTx) is a promising viable near-term solution to the shortage of hearts for clinical transplantation. In recent years, there has been a remarkable improvement in survival of heterotopic pig-to-nonhuman primate (NHP) CXTx [5-8], encouraging early success in orthotopic CXTx (oCXTx) [9-11] and advances in lifesupporting renal xenotransplantation $(\mathrm{RXTx})[12,13]$. These results validate the physiological compatibility of porcine organs, at least in NHPs, and suggest that clinical CXTx may soon be applicable if oCXTx can attain similar improvements in survival as RXTx. In this review, we examine developments in immune suppression, porcine donor genetics, preclinical transplants, and infectious disease issues and discuss requirements for clinical CXTx.

To justify a clinical xenotransplantation (XTx) program, it is necessary to demonstrate transplant efficacy in clinically relevant animal models. The International Society of Heart Lung Transplantation (ISHLT) has suggested that a prospectively defined series of life-supporting cardiac xenotransplants in NHPs, using predefined immune suppression, with " $60 \%$ survival at 3 months with a minimum of 10 animals surviving for this period," would be sufficient to consider a clinical trial [14]. The recent survival achieved after heterotopic CXTx (hCXTx), in excess of 2 years and with a median survival of 298 days [8], suggests that this goal may be attainable. While the ISHLT recommendation has become a de facto guideline for researchers in 
TABLE 1: The longest (median) reported heterotopic cardiac xenograft survival as a function of donor genetics and immune suppression.

\begin{tabular}{lcccc}
\hline Donor & \multicolumn{2}{c}{ Earlier immune suppression } & \multicolumn{2}{c}{ Costimulation blockade } \\
& CsA/CyP/steroid & ATG/CD20/tacrolimus/sirolimus & $\begin{array}{c}\text { ATG/LoCD2b/CVF/ } \\
\text { anti-CD154/MMF }\end{array}$ & nTG/anti-CD40, \\
CD20/CVF/MMF
\end{tabular}

n.r.: none reported. ${ }^{\S}$ Soluble CR1 to block complement activation. ${ }^{\ddagger}$ Cobra venom factor at $0.25-0.5$ mg/kg prior to surgery and $0.1-0.5$ mg/kg every $1-4$ days thereafter. *Included use of alpha-Gal polymer GAS914 [127] or Nex1285 [128]. ' Immune suppression included anti-CD20 (Rituximab) B-cell depletion. ${ }^{\Delta}$ hDAF (human CD55) minigene [129]. ${ }^{\nabla}$ A murine $\mathrm{H}-2 \mathrm{~Kb}$ regulated human CD55 transgene [77]. ${ }^{*} \mathrm{hCD} 46$ transgene based on $60 \mathrm{~kb}$ human genomic CD46 DNA [130]. ' hCD46 transgene based on a human CD46 minigene [131].

the field, the FDA, responsible for regulating XTx, has not officially endorsed these specific criteria, making early interactions with regulators essential to advance a clinical study.

The most prominent variables which contribute to XTx efficacy are likely to be immune suppression and donor genetics, but recipient species [15], viral status [16-18], the level of preexisting anti-pig antibody [13], prophylactic antiviral and antibacterial therapy $[19,20]$, and postoperative care [21] also significantly contribute to graft survival. The relative contribution of immune suppression and donor genetics is incompletely understood in a field undergoing rapidly evolving experimental changes in both components. Past reviews of the state-of-the art in XTx [22-24] and recent publications $[8,25]$ are useful for gaining an appreciation for the breadth of changes in XTx organ survival over the last 20 years. Table 1, showing the longest reported (and median) graft survival for various donor genetics under four broadly defined immune suppression regimens, summarizes these advances for CXTx. Not surprisingly, all combinations of donor genetics and immune suppression have not been reported, as perceived advances in donor genetics were seldom tested with reduced or previous immune suppression techniques.

\section{Earlier Immune Suppression}

Early hCXTx studies using Gal-positive (WT) donor hearts focused on preventing hyperacute rejection and often used immune suppression based largely on cyclophosphamide, cyclosporine, and steroids (CCS) [26]. Maximal hCXTx survival of 32 days was achieved in pig-to-cynomolgus monkey transplants using soluble CR1 to block systemic complement activation [27]. Comparable results were also reported after pig-to-baboon hCXTx using cobra venom factor (CVF) to consume complement [28]. CCS immune suppression was also used in early hCXTx studies using transgenic donors expressing human complement regulatory proteins (hCRPs). The longest reported survival was 99 days (median 26 days) using hDAF transgenic hearts [29]. These results demonstrated that expression of hCRPs was sufficient to abrogate the need for systemic complement inhibition, but was not sufficient to prevent an induced antibody response and antibody-mediated rejection (AMR). When anti-Gal antibody was blocked in vivo using a Gal polymer, more consistent graft survival, median 35 days, was reported in pig-to-cynomolgus monkey hCXTx [30]. This study appears to be the first to detect an induced non-Gal antibody associated with xenograft rejection. These early CCS regimens were often poorly tolerated due to the narrow therapeutic index for cyclophosphamide.

An alternative immune suppression strategy based on induction with ATG and Rituximab and using tacrolimus and sirolimus maintenance immune suppression was used in WT;hCRP, GGTA-1 $\alpha$-galactosyltransferase-deficient pigs (GTKO), and GTKO;hCRP transplants. The studies with WT;hCRP donors involved the largest series of transplants $(n=63)$ using a Gal polymer to test the effects of systemic anticoagulation and immune suppression on graft survival [31]. At moderate tacrolimus and sirolimus maintenance levels, hCXTx graft survival of up to 109 days (median survival 20 days) was achieved, similar to earlier results using CCS immune suppression. At higher levels of maintenance immune suppression [32,33], maximal survival was improved (139 days) with more consistent and prolonged median survival of 96 days. This was the first instance of median cardiac xenograft survival in excess of 3 months. In these WT;hCRP studies, anti-Gal-mediated rejection was minimized and graft rejection was associated with non-Gal antibody. Moreover, testing three distinct, tightly controlled clinical anticoagulation therapies did not improve graft survival or affect the histology of graft rejection, indicating no strict requirement for, or benefit from, systemic anticoagulation. Using GTKO or GTKO;hCRP donors with moderate tacrolimus and sirolimus, immune suppression achieved comparable survival to earlier WT;hCRP transplants using Gal polymers, indicating that the adoption of GTKO donors obviated the need for specific therapy to control anti-Gal antibody and suggested that graft survival was limited by the effects of non-Gal antibody [34]. Also, the three-month median survival achieved in these hCXTx 
studies made the conversion to oCXTx the appropriate model of choice moving forward.

\section{Costimulation Blockade Immune Suppression}

The more recent form of immune suppression utilized in hCXTx is costimulation blockade, primarily directed at the CD154 (CD40 ligand) and CD40 secondary signaling pathway, to block Th2 cell help for B cell activation. Antibody to CD154, originally shown to suppress allograft rejection [35], has been used extensively in pig-to-NHP hCXTx with donor organs ranging from WT;hCRP to GTKO;hCRP donors (Table 1). This immune suppression regimen was complex, including lymphocyte depletion with ATG and LoCD2b antibody, complement inhibition with CVF and steroids, and chronic postoperative immune suppression with mycophenolate mofetil and anti-CD154 antibody (5c8), and usually supplemented with a range of anticoagulant therapies. Early versions of this protocol also included pretransplant thymic irradiation [36] and used Gal polymers. With WT;hCRP donor hearts, maximal hCXTx organ survival was 139 days with a median of 23 days. More consistent organ survival was achieved (median 78 days) by transplantation of GTKO donor hearts into recipient baboons with little or no detected preformed non-Gal antibody [37]. In both instances, there was no apparent induction of circulating Gal or non-Gal antibody although the histology of the graft at explant, showing vascular antibody and complement deposition, was consistent with AMR. Importantly, antiCD154 costimulation blocking regimens often reported complications with thrombocytopenia, consumptive coagulopathy (CC), and systemic inflammation which contributed to recipient loss $[38,39]$. With GTKO;CD46 donor hearts, 236-day maximal graft survival (median survival 71 days) was achieved using a modified anti-CD154 protocol which included Rituximab induction to deplete B-cells [5]. Intensive postoperative monitoring in this study also likely contributed to prolonging graft survival. Explanted grafts showed evidence of ongoing humoral rejection; however, the authors indicated that survival was largely limited by nonimmune model-related issues for managing the recipient animals and recurrent thrombocytopenia, ascribed to the use of anti-CD154. The continued use of CVF, even with GTKO;CD46 donor organs, also contributes to systemic coagulation perturbations [40], precluding its clinical use.

Systemic thrombocytopenia and CC associated with antiCD154 binding to activated platelets are well known, and significant efforts have been made in XTx models to find an effective substitute costimulation blockade regimen [41, 42]. Recent studies demonstrate that chronic administration of an anti-CD40 blocking antibody (2C10R4), substituting for anti-CD154, leads to prolonged hCXTx survival [25]. In the initial report, anti-CD40 (2C10R4), administered for just 60 days posttransplant, achieved maximal GTKO;hCRP hCXTx survival of 149 days and median survival of 84 days [25]. Graft survival appeared to be limited by the dosage and duration of immune suppression as withdrawal of anti-CD40 therapy resulted in a marked rise in antibody titer and xenograft rejection. Importantly, substituting anti-CD40 antibody for anti-CD154 moderated complications of thrombocytopenia and consumptive coagulopathy, which may also have contributed to improved graft survival. Using a higher dosage of anti-CD40 (2C10R4), administered for longer, resulted in longer survival of GTKO;hCRP donor hearts expressing human thrombomodulin (TBM) with maximal survival of 945 days (median survival 298 days) [8]. These outcomes likely underestimate survival as anti-CD40 therapy was reduced in 2 of 5 recipients after 100 days and was reduced for two other recipients after 1 year. In these latter recipients, anti-CD40 therapy was eventually withdrawn at 560 and 861 days posttransplant. In each case, reduction/cessation of anti-CD40 therapy resulted in induction of non-Gal IgM and IgG antibody with eventual graft rejection. Despite persistent vascular expression of human TBM, the histology of explanted rejected hearts exhibited features typical of xenograft rejection including thrombotic microangiopathy, vasculitis, intravascular thrombosis, and myocardial necrosis with little evidence of lymphocytic infiltration suggesting limited impact of the TBM addition. Recipients also received CVF, heparin, and aspirin.

The new anti-CD40-based costimulation blocking regimen appears to have achieved a level of humoral and cellular immune suppression which, for the duration that it is provided, blocks non-Gal AMR, with graft survival now measured in years. This appears to surmount a major obstacle to clinical XTx. While the current anti-CD40 (2C10R4) is a mouse/rhesus chimeric IgG4 antibody which would not be suitable for use in humans, it is reportedly being humanized [6]. There are also several other humanized anti-CD40 blocking antibodies under various levels of development (Table 2), suggesting there may soon be a clear path forward for immune suppression, using only approved therapies, to support clinical CXTx.

\section{Donor Genetics}

Genetic engineering of the donor pig is a cornerstone of XTx as it enhances organ survival and function, while reducing the need for systemic therapies in the recipient. The pace of genetic manipulation of the pig genome has significantly increased with the introduction of somatic cell nuclear transfer and sequence-directed nucleases [43, 44]. This proliferation currently outstrips the pace of analysis in pig-to-NHP transplants. There are now dozens of reported gene additions or deletions $[45,46]$ with suggestions that donor animals with 5 or more genetic alterations affecting complement regulation, antigen reduction, haemostatic incompatibilities, coagulation dysfunction, suppression of inflammation, adaptive T-cell immunity, and endogenous retrovirus infectious risks may be required for clinical XTx [47]. In the search for an "ideal" donor, the simultaneous introduction of multiple genetic modifications, without appropriately controlled experiments, may obfuscate their function, as well as introduce unnecessary complications. A consistent strategic approach to developing and testing new donor genetics would accelerate the application of clinical CXTx. Moreover, the accumulation of multiple gene modifications complicates 
TABLe 2: Anti-CD40 antibodies in clinical development.

\begin{tabular}{lccr}
\hline Antibody & Company & Status & Trial ID \\
\hline \multirow{2}{*}{ SGN-40 } & & Phase 1 multiple myeloma & NCT00079716 \\
& Seattle Genetics Inc. & Phase 2 B-cell lymphoma & NCT00435916 \\
NCT00283101 & Phase 1/2 chronic lymphocytic leukemia & NCT01780844 \\
ASKP 1240 & Astellas & Phase 2 renal Tx phase 2 plaque & NCT01585233 \\
\hline & & Psoriasis & NCT00108108 \\
HCD122 & Novartis & Phase 1, chronic lymphocytic leukemia & NCT00231166 \\
& & Phase 2 multiple myeloma & NCT01275209 \\
Chi Lob 7/4 & Phase 2 follicular lymphoma & NCT00670592 \\
BG9588 & Cancer Res UK & Phase 1/2 Hodgkin's and non-Hodgkin's lymphoma & NCT01561911 \\
& NIDDK & Phase 1 cancer malignancies & NCT00001857 \\
\hline & NIAMS & Phase 2 renal Tx & NCT00001789 \\
\hline
\end{tabular}

donor breeding programs to the point that somatic cell cloning may be required to maintain the genetic profile. This will increase cost and may limit the production of donor animals for preclinical studies, the results of which have already been frequently compromised by small group sizes.

Donor genetic modifications have focused on four main categories, antigen reduction [48], thromboregulation [49], immune suppression, and infectious disease [50]. Two additional non-Gal glycan antigens have been identified, $\mathrm{N}$-glycolylneuraminic acid- (Neu5Gc-) modified oligosaccharides $[51,52]$ and the glycan product synthesized by porcine beta 1,4-N-acetylgalactosamine transferase-2 (B4GALNT2) [53]. Humans do not synthesize Neu5Gc due to a mutation in the CMP-N-acetylneuraminic acid hydroxylase gene (CMAH), but they do produce an array of antibodies which show Neu5Gc-dependent reactivity to sialylated oligosaccharides. These anti-Neu5Gc antibodies are noted for their role in serum sickness in patients treated with animal sera [54]. The B4GALNT2 gene catalyses the terminal addition of $\mathrm{N}$-acetylgalactosamine (GalNAc) to a sialic acid-modified lactosamine acceptor producing GalNAc $\beta 4$ [Neu5Ac $\alpha 2,3]$ Gal $\beta 4 \mathrm{GlcNAc} \beta 3 \mathrm{Gal}$, the SDa blood group antigen. This is an immunogenic glycan in pig-to-NHP CXTx [55]. Humans are known to produce low levels of IgM which bind the polyagglutinable human SDa blood group [56-59]. Targeted mutations affecting the porcine CMAH and B4GALNT2 genes have been made and combined with the GTKO mutation [48, 60-62]. These three mutations together minimized human IgM and IgG binding to porcine cells in over $90 \%$ of human serum samples [48] and reduced both IgM and IgG reactivity to background levels in $30 \%$ of allosensitized wait-listed renal transplant candidates [60]. Pig-to-NHP CXTx using organs with these mutations has not yet been reported. Minimizing tissue immunogenicity would appear to have obvious clinical benefit but demonstrating this in preclinical transplants will be difficult as NHPs do not produce anti-Neu5Gc antibody. Additionally, induction therapy with ATG, part of the current costimulation blocking immune suppression, may induce an anti-Neu5Gc antibody response in humans [63], which is not apparent in NHPs. This induced response could sensitize recipients and compromise Neu5Gc-positive donor organs in clinical CXTx. This suggests that new large animal transplant models, using $\mathrm{CMAH}$-deficient recipients, may be required to test the pathogenicity of anti-Neu5Gc antibody and optimize the use of biological agents for immune suppression. Despite these issues, genetic engineering directed at reducing the antigenicity of porcine tissue is likely to significantly impact clinical CXTx.

Interest in transgenic augmentation of thromboregulation stems primarily from recognition of molecular incompatibilities between porcine and human TBM [64]. Secondarily, immune-independent recipient and donor cell-to-cell interactions have been described in vitro which are proposed to contribute to donor endothelial cell activation $[65,66]$, systemic haemostatic dysfunction, and CC [67-69]. Several groups have reported production of human TBM transgenic pigs [70-72], and in vitro analysis of porcine endothelial cells expressing human TBM shows that it alleviates the molecular incompatibility with efficient production of activated human protein C $[64,73]$. A limited number of pig-to-NHP CXTx studies with human TBM expressing donor organs have been reported $[8,74]$. The impact of human TBM expression cannot be determined from these studies as their designs lacked controls without TBM, included chronic systemic heparin administration, and had no direct measure of human TBM function. Transgenic expression of other key components which affect haemostasis, CD39, CD47, TFPI, and EPCR, has also been reported $[12,75]$.

The common donor modifications, transgenic expression of hCRPs, and the GTKO mutation have been thoroughly analysed and validated. Physiological or supraphysiological vascular expression of one or more human complement regulatory genes, CD59, CD55, or CD46, establishes an intrinsic barrier which regulates the complement cascade [76-78], reduces the incidence of hyperacute rejection, and limits complement-mediated injury [15]. Likewise, the significance of anti-Gal antibody and benefit of targeted mutation of the porcine $\alpha$-galactosyltransferase locus [79-81] have been extensively documented in in vitro [82] and in vivo studies in both pig-to-NHP [83-86] and GTKO mouse models [87-91]. The combination of hCRP and GTKO donor modifications has also been specifically examined and demonstrated to be beneficial, preventing rare hyperacute 
GTKO hCXTx rejection and early immune injury [34, 92]. This basic genetic background, GTKO;hCRP, represents, in our judgement, the current, proven starting base for any clinical study. Additional CMAH- and B4GALNT2directed antigen reduction would appear to be a beneficial clinical priority.

\section{Orthotopic CXTx and Perioperative Graft Function}

The vast bulk of CXTx studies have to date utilized an abdominal non-life-supporting heterotopic transplant model where the graft is contractile but does not support the recipient's circulation. In comparison, there have been a limited number of oCXTx studies [9-11, 93-99]. These studies, predominantly using WT;hCRP donors, report healthy recipient survival up to 57 days [46]. In this difficult model, recipient death is often due to postoperative management limitations, with explanted hearts often showing little histologic evidence of significant rejection. These studies, which could not yet utilize the most recent highly successful costimulation blockade immune suppression, clearly indicate that porcine hearts can provide life-sustaining and adequate circulation to NHPs and suggest that the efficacy of oCXTx is not intrinsically limited by cardiac function but by immune rejection and postoperative management. To demonstrate life-supporting oCXTx in a preclinical NHP model, consistent with the ISHLT guidelines, will require not only effective immune suppression and appropriate donor genetics but also substantial clinical level resources, expertise, and postoperative management.

Orthotopic CXTx studies also unmasked a potential impediment to clinical CXTx which was not apparent in hCXTx studies. Every research group that has performed oCXTx has reported variable perioperative mortality ranging from 40 to $60 \%$ within the first 48 hours. Xenograft failure in this time period was not due to hyperacute rejection as the explanted hearts show vascular antibody deposition but otherwise normal myocardial histology [100]. Instead, early graft failure was associated with primary organ dysfunction. We have called this phenomenon perioperative cardiac xenograft dysfunction (PCXD) which at this high frequency currently represents a significant barrier to clinical CXTx. Our ongoing studies suggest that PCXD is similar to ischemia reperfusion injury or cardiac stunning. We find that PCXD can be modulated with a preconditioning regimen to reduce circulating antibody, B-cells, and plasma cells prior to transplant, coupled with improved organ preservation [101]. In recipients which survive beyond 48 hours, PCXD is less evident and echocardiographic analysis indicates that PCXD is completely reversible showing that the normal cardiac reparative processes function across the XTx barrier [11]. Intrathoracic heterotopic cardiac transplantation, where both the donor and recipient hearts contribute to the circulation, was successfully used in early allotransplantation when techniques for donor organ preservation were being optimized. Preclinical intrathoracic heterotopic cardiac xenotransplantation studies, although complex, potentially offer a unique opportunity to study the aetiology and recovery from PCXD [102, 103]. Genetic engineering approaches may also have the potential to mitigate PCXD, for example, by providing high levels of CD39 expression [75] or reducing sodium hydrogen ion exchange activity [104].

\section{Diagnosis and Treatment of Rejection}

The ability to diagnose and treat rejection is a key component of clinical transplantation. The ISHLT has developed pathologic grades (pAMR1-3) of immunopathologic features of endomyocardial biopsies which along with graft dysfunction and levels of donor-specific antibody are used for the diagnosis AMR in cardiac allotransplantation [105]. The most severe pathology (pAMR3), associated with significant graft dysfunction and poor clinical outcomes, can be treated with a combination of increased and optimized immune suppression, depletion of circulating antibody, and IVIg. More aggressive salvage therapies may also include B-cell and plasma cell depletion and complement inhibition $[105,106]$. In CXTx, there are few studies which have attempted to diagnose and treat presumptive rejection episodes, most of these after hCXTx [9, 25, 33, 107-109]. Putative rejection episodes were diagnosed based on biochemical markers (troponin T, AST), graft contractility, telemetric measures of cardiac performance, and echocardiography. Serial biopsies after oCXTx will likely be applicable for diagnosis of rejection [109-111], but the difficulty of obtaining endomyocardial biopsies in NHPs has limited their exploration in animal models. When presumptive rejection episodes were treated using steroids, or steroids and ATG, there was no evidence for reversal of rejection, and, unsurprisingly, in some instances, excessive antirejection therapy increased the frequency of infectious complications $[9,25]$. Effective therapies to reverse AMR in XTx remain to be fully explored.

Based on the high frequency of AMR, the wide diversity of potential polymorphic porcine peptides and the chronic detection of vascular antibody deposition in GTKO donor hearts, it is necessary to establish methods for early diagnosis and effective treatment. It appears that anti-CD40-based immune suppression, which is likely to be used in clinical CXTx, relies heavily on effective costimulation blockade, as withdrawal of anti-CD40 therapy has resulted in the induction of non-Gal IgM and IgG [8]. This has at least two potential consequences. Firstly, costimulation blockade complicates the use of plasmapheresis, commonly used to treat AMR, as it would remove both therapeutic and pathogenic antibodies. Secondly, chronic dosing with biological therapeutics risks the development of anti-antibody immune responses $[112,113]$. While the frequency of this response is difficult to estimate and cannot be safely extrapolated from the results reported for other antibody-based therapeutics, in the context of clinical CXTx, an anti-anti-CD40 response would be potentially serious. Few clinical methods can reverse antibody-mediated heart rejection so it will be important, prior to clinical CXTx, to develop and test, to as great a degree as possible, xenospecific therapies for detecting and treating AMR using a pig-to-NHP transplant model. This may include alternative versions of anti-CD40 (Table 2), alternative costimulation strategies [13], total lymphoid 
radiation [114], or current antibody reduction therapies $[105,106]$. Ideally, such a study would be performed using CXTx, but life-supporting kidney XTx, with well-known physiological markers for organ function, maybe a more pragmatic solution as ongoing kidney rejection will not result in rapid recipient death. What is clear to investigators with experience in oCXTx is that the NHP model plays a critical role in progressing to clinical application, but has intrinsic limitations with particular regard to recipient management.

\section{Infectious Disease Issues}

Complete knowledge and risk-free application of clinical CXTx, as with most major advances in medicine, retains elements of uncertainty. The potential for disease transmission has been a significant concern for clinical XTx [115]. Concern has been expressed about the potential of porcine endogenous retroviruses (PERV) to emerge in XTx recipients, infect patient tissues, and adapt to humans [116]. Since this potential was identified, molecular and immunologic assays to monitor PERV infection have been developed [117], and significant advances have been made in mapping PERV proviral sites $[118,119]$ and understanding the basic biology of PERV infection [120]. Several clinical studies of patients exposed to porcine tissues [121-124] or in NHP XTx recipients [125] have also failed to detect PERV infection. The generation of high titer human-trophic PERV requires the recombination of relatively rare PERV-C proviral sequences with more common PERV-A. Selective breeding can be used to eliminate PERV-C from donor pigs [126]. Alternatively, nuclease-directed mutation of the PERV pol gene has been shown to induce widespread PERV proviral deletions [50], but this may be unnecessary if PERV-C is eliminated by selective breeding. While diligent monitoring of PERV infection in XTx recipients is prudent, the apparent risk presented by PERV appears to be small and is unlikely to delay clinical CXTx. Aside from endogenous retrovirus, specific pathogen-free (SPF) donor pig facilities have been produced and populated with caesarean-derived piglets. Some of these sites have been operational and breeding pigs for many years demonstrating the feasibility to routinely produce donor pigs with exceptionally high health standards.

\section{Conclusion}

It is clear that cardiac and renal XTx can benefit patients in need of organ replacement. If continued studies as outlined above are performed, we are optimistic that this technology will soon be ready for clinical testing. These remaining key preclinical studies are required to ensure the efficacy and safety of clinical CXTx. Principally a life-supporting preclinical oCXTx study in NHPs must be performed to demonstrate acceptable perioperative and postoperative recipient survival. This study should optimize organ preservation and utilize immune suppression based on anti-CD40 costimulation blockade. To meet ISHLT suggested standards [14], this study, involving at least 16 CXTx recipients, will require significant financial resources, an infrastructure to simultaneously maintain multiple CXTx postoperative recipients and a dedicated team of clinicians, veterinarians, scientists, and animal technologists. Donor organs should minimally contain GTKO;hCRP genetics, likely with additional antigen reduction of CMAH-KO and B4GALNT2KO. Clinical use of additional genetic modifications should be founded on further rigorous preclinical testing in NHPs to demonstrate their utility, which, in the case of CMAH, will likely require testing in an immunologically appropriate $\mathrm{CMAH}-\mathrm{KO}$ large animal transplant model. As well as achieving adequate perioperative and postoperative recipient survival, such a preclinical study should allow for organ rejection. This rejection study, while unlikely to fully predict the clinical immune response, will suggest essential xenospecific assays and therapies to reverse rejection which can be further refined during clinical CXTx.

Genetic engineering has significantly improved CXTx organ survival but ongoing creation of new genetics, in pursuit of the perfect donor, has the potential to delay clinical studies. We believe the initial clinical studies will rely primarily on known systemic immune suppression and genetics and that further optimization of donor genetics is best pursued in response to identified, researched clinical immune and physiological requirements.

Dependent on the results of these prospective preclinical oCXTx studies in NHPs, it appears to us that the era of clinical CXTx is approaching. The scientific path forward is demanding but well defined; however, the complexity of any clinical XTx program, including the heart, presents a substantial and unique set of regulatory challenges which need to be addressed expeditiously to avoid delaying the realization of clinical use.

\section{Abbreviations}

AMR: Antibody-mediated rejection

B4GALNT2: Beta 1,4-N-acetylgalactosamine transferase-2

CC: $\quad$ Consumptive coagulopathy

CCS: $\quad$ Cyclophosphamide, cyclosporine, and steroids

CMAH: $\quad$ CMP-N-acetylneuraminic acid hydroxylase

CVF: Cobra venom factor

CXTx: Cardiac xenotransplantation

GTKO: Alpha-galactosyltransferase (GGTA_1) mutant

hCRPs: Human complement regulatory proteins

hCXTx: Heterotopic CXTx

ISHLT: International Society of Heart and Lung

Transplantation

Neu5Gc: N-Glycolylneuraminic acid

NHP: $\quad$ Nonhuman primate

oCXTx: $\quad$ Orthotopic CXTx

PCXD: Perioperative cardiac xenograft dysfunction

PERV: $\quad$ Porcine endogenous retroviruses

RXTx: Renal xenotransplantation

SPF: $\quad$ Specific pathogen free

TBM: $\quad$ Thrombomodulin

WT: Gal-positive pig

XTx: Xenotransplantation. 


\section{Conflicts of Interest}

The authors declare that there is no conflict of interest regarding the publication of this paper.

\section{Acknowledgments}

This study is supported by the NIH Grant AI66310, the MRC Grant MR L013193, and the NIHR UCL Biomedical Research Centre.

\section{References}

[1] Writing Group Members, D. Mozaffarian, E. J. Benjamin et al., "Heart disease and stroke statistics-2016 update: a report from the American Heart Association," Circulation, vol. 133, no. 4, pp. e38-e360, 2016.

[2] E. A. Rose, A. C. Gelijns, A. J. Moskowitz et al., "Long-term use of a left ventricular assist device for end-stage heart failure," The New England Journal of Medicine, vol. 345, no. 20, pp. 1435-1443, 2001.

[3] A. Carpentier, C. Latrémouille, B. Cholley et al., "First clinical use of a bioprosthetic total artificial heart: report of two cases," The Lancet, vol. 386, no. 10003, pp. 1556-1563, 2015.

[4] J. K. Kirklin, D. C. Naftel, R. L. Kormos et al., "Fifth INTERMACS annual report: risk factor analysis from more than 6,000 mechanical circulatory support patients," The Journal of Heart and Lung Transplantation, vol. 32, no. 2, pp. 141156, 2013.

[5] M. M. Mohiuddin, P. C. Corcoran, A. K. Singh et al., "B-cell depletion extends the survival of GTKO.hCD46Tg pig heart xenografts in baboons for up to 8 months," American Journal of Transplantation, vol. 12, no. 3, pp. 763-771, 2012.

[6] M. M. Mohiuddin, A. K. Singh, P. C. Corcoran et al., "Genetically engineered pigs and target-specific immunomodulation provide significant graft survival and hope for clinical cardiac xenotransplantation," The Journal of Thoracic and Cardiovascular Surgery, vol. 148, no. 3, pp. 1106-1113, 2014.

[7] M. M. Mohiuddin, A. K. Singh, P. C. Corcoran et al., "Oneyear heterotopic cardiac xenograft survival in a pig to baboon model," American Journal of Transplantation, vol. 14, no. 2, pp. 488-489, 2014.

[8] M. M. Mohiuddin, A. K. Singh, P. C. Corcoran et al., "Chimeric 2C10R4 anti-CD40 antibody therapy is critical for long-term survival of GTKO.hCD46.hTBM pig-to-primate cardiac xenograft," Nature Communications, vol. 7, article 11138, 2016.

[9] G. W. Byrne, Z. Du, Z. Sun, Y. W. Asmann, and C. G. McGregor, "Changes in cardiac gene expression after pigto-primate orthotopic xenotransplantation," Xenotransplantation, vol. 18, no. 1, pp. 14-27, 2011.

[10] C. G. A. McGregor, G. W. Byrne, M. Vlasin et al., "Cardiac function after preclinical orthotopic cardiac xenotransplantation," American Journal of Transplantation, vol. 9, no. S2, p. 380, 2009.

[11] C. G. A. McGregor, W. R. Davies, K. Oi et al., "Recovery of cardiac function after pig-to-primate orthotopic heart transplant. (Abstr 98)," American Journal of Transplantation, vol. 8, no. s2, pp. 205-206, 2008.

[12] H. Iwase, H. Hara, M. Ezzelarab et al., "Immunological and physiological observations in baboons with life-supporting genetically engineered pig kidney grafts," Xenotransplantation, vol. 24, no. 2, 2017.

[13] L. Higginbotham, D. Mathews, C. A. Breeden et al., "Pretransplant antibody screening and anti-CD154 costimulation blockade promote long-term xenograft survival in a pig-toprimate kidney transplant model," Xenotransplantation, vol. 22, no. 3, pp. 221-230, 2015.

[14] D. K. Cooper, A. M. Keogh, J. Brink et al., "Report of the Xenotransplantation Advisory Committee of the International Society for Heart and Lung Transplantation: the present status of xenotransplantation and its potential role in the treatment of end-stage cardiac and pulmonary diseases," The Journal of Heart and Lung Transplantation, vol. 19, no. 12, pp. 1125-1165, 2000.

[15] H.-J. Schuurman, G. Pino-Chavez, M. J. Phillips, L. Thomas, D. J. G. White, and E. Cozzi, "Incidence of hyperacute rejection in pig-to-primate transplantation using organs from hDAF-transgenic donors," Transplantation, vol. 73, no. 7, pp. 1146-1151, 2002.

[16] N. J. Mueller, K. Kuwaki, F. J. Dor et al., "Reduction of consumptive coagulopathy using porcine cytomegalovirusfree cardiac porcine grafts in pig-to-primate xenotransplantation," Transplantation, vol. 78, no. 10, pp. 1449-1453, 2004.

[17] K. Yamada, M. Tasaki, M. Sekijima et al., "Porcine cytomegalovirus infection is associated with early rejection of kidney grafts in a pig to baboon xenotransplantation model," Transplantation, vol. 98, no. 4, pp. 411-418, 2014.

[18] H. Zhou, H. Iwase, R. F. Wolf et al., "Are there advantages in the use of specific pathogen-free baboons in pig organ xenotransplantation models?," Xenotransplantation, vol. 21, no. 3, pp. 287-290, 2014.

[19] J. A. Fishman, "Infection in xenotransplantation," Journal of Cardiac Surgery, vol. 16, no. 5, pp. 363-373, 2001.

[20] S. S. Teotia, R. C. Walker, J. M. Schirmer et al., "Prevention, detection, and management of early bacterial and fungal infections in a preclinical cardiac xenotransplantation model that achieves prolonged survival," Xenotransplantation, vol. 12, no. 2, pp. 127-133, 2005.

[21] P. C. Corcoran, K. A. Horvath, A. K. Singh et al., "Surgical and nonsurgical complications of a pig to baboon heterotopic heart transplantation model," Transplantation Proceedings, vol. 42, no. 6, pp. 2149-2151, 2010.

[22] D. Lambrigts, D. H. Sachs, and D. K. C. Cooper, "Discordant organ xenotransplantation in primates: world experience and current status," Transplantation, vol. 66, no. 5, pp. 547-561, 1998.

[23] B. Ekser, P. Rigotti, B. Gridelli, and D. K. Cooper, "Xenotransplantation of solid organs in the pig-to-primate model," Transplant Immunology, vol. 21, no. 2, pp. 87-92, 2009.

[24] D. K. Cooper, V. Satyananda, B. Ekser et al., "Progress in pigto-nonhuman primate transplantation models (1998-2013): a comprehensive review of the literature," Xenotransplantation, vol. 21, no. 5, pp. 397-419, 2014.

[25] M. M. Mohiuddin, A. K. Singh, P. C. Corcoran et al., "Role of anti-CD40 antibody-mediated costimulation blockade on non-Gal antibody production and heterotopic cardiac xenograft survival in a GTKO.hCD46Tg pig-to-baboon model," Xenotransplantation, vol. 21, no. 1, pp. 35-45, 2014.

[26] J. L. Platt, S. S. Lin, and C. G. A. McGregor, "Acute vascular rejection,” Xenotransplantation, vol. 5, no. 3, pp. 169-175, 1998. 
[27] E. A. Davis, S. K. Pruitt, P. S. Greene et al., "Inhibition of complement, evoked antibody, and cellular response prevents rejection of pig-to-primate cardiac xenografts," Transplantation, vol. 62, no. 7, pp. 1018-1023, 1996.

[28] T. Kobayashi, S. Taniguchi, F. A. Neethling et al., "Delayed xenograft rejection of pig-to-baboon cardiac transplants after cobra venom factor therapy," Transplantation, vol. 64, no. 9, pp. 1255-1261, 1997.

[29] F. N. K. Bhatti, M. Schmoeckel, A. Zaidi et al., "Three-month survival of HDAFF transgenic pig hearts transplanted into primates," Transplantation Proceedings, vol. 31, p. 958, 1999.

[30] T. T. Lam, R. Paniagua, G. Shivaram, H. J. Schuurman, D. C. Borie, and R. E. Morris, "Anti-non-Gal porcine endothelial cell antibodies in acute humoral xenograft rejection of hDAF-transgenic porcine hearts in cynomolgus monkeys," Xenotransplantation, vol. 11, no. 6, pp. 531-535, 2004.

[31] G. W. Byrne, W. R. Davies, K. Oi et al., "Increased immunosuppression, not anticoagulation, extends cardiac xenograft survival," Transplantation, vol. 82, no. 12, pp. 1787-1791, 2006.

[32] C. G. McGregor, W. R. Davies, K. Oi et al., "Cardiac xenotransplantation: recent preclinical progress with 3-month median survival," The Journal of Thoracic and Cardiovascular Surgery, vol. 130, no. 3, pp. 844.e1-851.e9, 2005.

[33] C. G. A. McGregor, S. S. Teotia, G. W. Byrne et al., "Cardiac xenotransplantation: progress toward the clinic," Transplantation, vol. 78, pp. 1569-1575, 2004.

[34] C. G. McGregor, D. Ricci, N. Miyagi et al., "Human CD55 expression blocks hyperacute rejection and restricts complement activation in Gal knockout cardiac xenografts," Transplantation, vol. 93, no. 7, pp. 686-692, 2012.

[35] A. D. Kirk, D. M. Harlan, N. N. Armstrong et al., "CTLA4-Ig and anti-CD40 ligand prevent renal allograft rejection in primates," PNAS, vol. 94, pp. 8789-8794, 1997.

[36] K. Kuwaki, C. Knosalla, F. J. Dor et al., "Suppression of natural and elicited antibodies in pig-to-baboon heart transplantation using a human anti-human CD154 mAbbased regimen," American Journal of Transplantation, vol. 4, no. 3, pp. 363-372, 2004.

[37] K. Kuwaki, Y. L. Tseng, F. J. Dor et al., "Heart transplantation in baboons using $\alpha 1,3$-galactosyltransferase gene-knockout pigs as donors: initial experience," Nature Medicine, vol. 11, no. 1, pp. 29-31, 2005.

[38] C. C. Lin, D. K. Cooper, and A. Dorling, "Coagulation dysregulation as a barrier to xenotransplantation in the primate," Transplant Immunology, vol. 21, no. 2, pp. 75-80, 2009.

[39] M. Ezzelarab, B. Garcia, A. Azimzadeh et al., "The innate immune response and activation of coagulation in $\alpha 1,3$ galactosyltransferase gene-knockout xenograft recipients," Transplantation, vol. 87, no. 6, pp. 805-812, 2009.

[40] G. W. Byrne, A. M. Azimzadeh, M. Ezzelarab et al., "Histopathologic insights into the mechanism of anti-non-Gal antibody-mediated pig cardiac xenograft rejection," Xenotransplantation, vol. 20, no. 5, pp. 292-307, 2013.

[41] P. Thompson, I. R. Badell, M. Lowe et al., "Alternative immunomodulatory strategies for xenotransplantation: CD40/154 pathway-sparing regimens promote xenograft survival," American Journal of Transplantation, vol. 12, no. 7, pp. 1765-1775, 2012.

[42] P. Thompson, K. Cardona, M. Russell et al., "CD40-specific costimulation blockade enhances neonatal porcine islet survival in nonhuman primates," American Journal of Transplantation, vol. 11, no. 5, pp. 947-957, 2011.

[43] P. Li, J. L. Estrada, C. Burlak, and A. J. Tector, "Biallelic knockout of the $\alpha-1,3$ galactosyltransferase gene in porcine liver-derived cells using zinc finger nucleases," The Journal of Surgical Research, vol. 181, no. 1, pp. e39-e45, 2013.

[44] P. Li, J. L. Estrada, C. Burlak et al., "Efficient generation of genetically distinct pigs in a single pregnancy using multiplexed single-guide RNA and carbohydrate selection," Xenotransplantation, vol. 22, no. 1, pp. 20-31, 2015.

[45] B. Ekser, M. Ezzelarab, H. Hara et al., "Clinical xenotransplantation: the next medical revolution?," Lancet, vol. 379, no. 9816, pp. 672-683, 2012.

[46] M. M. Mohiuddin, B. Reichart, G. W. Byrne, and C. G. McGregor, "Current status of pig heart xenotransplantation," International Journal of Surgery, vol. 23, Part B, pp. 234-239, 2015.

[47] D. K. Cooper, B. Ekser, J. Ramsoondar, C. Phelps, and D. Ayares, "The role of genetically engineered pigs in xenotransplantation research," The Journal of Pathology, vol. 238, no. 2, pp. 288-299, 2016.

[48] J. L. Estrada, G. Martens, P. Li et al., "Evaluation of human and non-human primate antibody binding to pig cells lacking GGTA1/CMAH/ $\beta 4$ GalNT2 genes," Xenotransplantation, vol. 22, no. 3, pp. 194-202, 2015.

[49] P. J. Cowan and S. C. Robson, "Progress towards overcoming coagulopathy and hemostatic dysfunction associated with xenotransplantation," International Journal of Surgery, vol. 23, Part B, pp. 296-300, 2015.

[50] L. Yang, M. Güell, D. Niu et al., "Genome-wide inactivation of porcine endogenous retroviruses (PERVs)," Science, vol. 350, no. 6264, pp. 1101-1104, 2015.

[51] H. H. Chou, H. Takematsu, S. Diaz et al., "A mutation in human CMP-sialic acid hydroxylase occurred after the Homo-Pan divergence," Proceedings of the National Academy of Sciences of the United States of America, vol. 95, no. 20, pp. 11751-11756, 1998.

[52] V. Padler-Karavani and A. Varki, "Potential impact of the non-human sialic acid $\mathrm{N}$-glycolylneuraminic acid on transplant rejection risk," Xenotransplantation, vol. 18, no. 1, pp. 1-5, 2011

[53] G. W. Byrne, Z. Du, P. Stalboerger, H. Kogelberg, and C. G. McGregor, "Cloning and expression of porcine $\beta 1,4 \mathrm{~N}$-acetylgalactosaminyl transferase encoding a new xenoreactive antigen," Xenotransplantation, vol. 21, no. 6, pp. 543-554, 2014

[54] H. Higashi, M. Naiki, S. Matuo, and K. Okouchi, "Antigen of "serum sickness" type of heterophile antibodies in human sera: identification as gangliosides with $\mathrm{N}$ glycolylneuraminic acid," Biochemical and Biophysical Research Communications, vol. 79, no. 2, pp. 388-395, 1977.

[55] G. W. Byrne, P. G. Stalboerger, Z. Du, T. R. Davis, and C. G. McGregor, "Identification of new carbohydrate and membrane protein antigens in cardiac xenotransplantation," Transplantation, vol. 91, no. 3, pp. 287-292, 2011.

[56] P. H. Renton, P. Howell, E. W. Ikin, C. M. Giles, and G. KLG, "Anti-Sd", a new blood group antibody," Vox Sanguinis, vol. 13, pp. 493-501, 1967.

[57] G. W. Bird and J. Wingham, "Cad(super Sd") in a British family with eastern connections: a note on the specificity of 
the Dolichos biflorus lectin," Journal of Immunogenetics, vol. 3, no. 5, pp. 297-302, 1976.

[58] M. D. Montiel, M. A. Krzewinski-Recchi, P. Delannoy, and A. Harduin-Lepers, "Molecular cloning, gene organization and expression of the human UDP-GalNAc:Neu5Acalpha2-3Galbeta- $\mathrm{R}$ beta1,4-N-acetylgalactosaminyltransferase responsible for the biosynthesis of the blood group Sda/ Cad antigen: evidence for an unusual extended cytoplasmic domain," The Biochemical Journal, vol. 373, Part 2, pp. 369-379, 2003.

[59] G. Danials, Human Blood Groups 3rd Edn, Chinchester, Wiley-Blackwell, 2013.

[60] G. R. Martens, L. M. Reyes, J. R. Butler et al., "Humoral reactivity of renal transplant-waitlisted patients to cells from GGTA1/CMAH/B4GalNT2, and SLA class I knockout pigs," Transplantation, vol. 101, no. 4, pp. e86-e92, 2017.

[61] J. R. Butler, G. R. Martens, J. L. Estrada et al., "Silencing porcine genes significantly reduces human-anti-pig cytotoxicity profiles: an alternative to direct complement regulation," Transgenic Research, vol. 25, no. 5, pp. 751-759, 2016.

[62] A. J. Lutz, P. Li, J. L. Estrada et al., "Double knockout pigs deficient in N-glycolylneuraminic acid and galactose $\alpha-1,3$ galactose reduce the humoral barrier to xenotransplantation," Xenotransplantation, vol. 20, no. 1, pp. 27-35, 2013.

[63] A. Salama, G. Evanno, N. Lim et al., "Anti-Gal and antiNeu5Gc responses in nonimmunosuppressed patients following treatment with rabbit anti-thymocyte polyclonal IgGs," Transplantation, vol. 101, pp. 2501-2507, 2017.

[64] C. W. Kopp, S. T. Grey, J. B. Siegel et al., "Expression of human thrombomodulin cofactor activity in porcine endothelial cells," Transplantation, vol. 66, no. 2, pp. 244-251, 1998.

[65] D. J. Goodman, M. Von Albertini, A. Willson, M. T. Millan, and F. H. Bach, "Direct activation of porcine endothelial cells by human natural killer cells," Transplantation, vol. 61, no. 5, pp. 763-771, 1996.

[66] S. Sheikh, R. Parhar, A. Kwaasi et al., "Alpha-gal-independent dual recognition and activation of xenogeneic endothelial cells and human naive natural killer cells," Transplantation, vol. 70, no. 6, pp. 917-928, 2000.

[67] H. Xu, F. Arnaud, D. K. Tadaki, L. C. Burkly, D. M. Harlan, and A. D. Kirk, "Human platelets activate porcine endothelial cells through a CD154-dependent pathway12," Transplantation, vol. 72, no. 11, pp. 1858-1861, 2001.

[68] C. C. Lin, D. Chen, J. H. McVey, D. K. Cooper, and A. Dorling, "Expression of tissue factor and initiation of clotting by human platelets and monocytes after incubation with porcine endothelial cells," Transplantation, vol. 86, no. 5, pp. 702-709, 2008.

[69] M. B. Ezzelarab and D. K. Cooper, "Systemic inflammation in xenograft recipients (SIXR): a new paradigm in pig-toprimate xenotransplantation?," International Journal of Surgery, vol. 23, Part B, pp. 301-305, 2015.

[70] B. Petersen, W. Ramackers, A. Tiede et al., "Pigs transgenic for human thrombomodulin have elevated production of activated protein C," Xenotransplantation, vol. 16, no. 6, pp. 486-495, 2009.

[71] S. Yazaki, M. Iwamoto, A. Onishi et al., "Production of cloned pigs expressing human thrombomodulin in endothelial cells," Xenotransplantation, vol. 19, no. 2, pp. 82-91, 2012.
[72] A. Wuensch, A. Baehr, A. K. Bongoni et al., "Regulatory sequences of the porcine THBD gene facilitate endothelialspecific expression of bioactive human thrombomodulin in single- and multitransgenic pigs," Transplantation, vol. 97, no. 2, pp. 138-147, 2014.

[73] Y. Miwa, K. Yamamoto, A. Onishi et al., "Potential value of human thrombomodulin and DAF expression for coagulation control in pig-to-human xenotransplantation," Xenotransplantation, vol. 17, no. 1, pp. 26-37, 2010.

[74] H. Iwase, B. Ekser, V. Satyananda et al., "Pig-to-baboon heterotopic heart transplantation - exploratory preliminary experience with pigs transgenic for human thrombomodulin and comparison of three costimulation blockade-based regimens," Xenotransplantation, vol. 22, no. 3, pp. 211-220, 2015.

[75] D. G. Wheeler, M. E. Joseph, S. D. Mahamud et al., "Transgenic swine: expression of human CD39 protects against myocardial injury," Journal of Molecular and Cellular Cardiology, vol. 52, no. 5, pp. 958-961, 2012.

[76] K. R. McCurry, D. L. Kooyman, L. E. Diamond, G. W. Byrne, J. S. Logan, and J. L. Platt, "Transgenic expression of human complement regulatory proteins in mice results in diminished complement deposition during organ xenoperfusion," Transplantation, vol. 59, no. 8, pp. 1177-1182, 1995.

[77] G. W. Byrne, K. R. McCurry, M. J. Martin, S. M. McClellan, J. L. Platt, and J. S. Logan, "Transgenic pigs expressing human CD59 and decay-accelerating factor produce an intrinsic barrier to complement-mediated damage," Transplantation, vol. 63, no. 1, pp. 149-155, 1997.

[78] E. Cozzi, N. Yannoutsos, G. A. Langford, G. Pino-Chavez, J. Wallwork, and D. J. G. White, "Effect of transgenic expression of human decay-accelerating factor on the inhibition of hyperacute rejection of pig organs," in Xenotransplantation, D. K. C. Cooper, E. Kemp, J. L. Platt and D. J. G. White, Eds., Springer-Verlag, Heidelberg, 1997.

[79] M. Diswall, J. Angstrom, H. J. Schuurman, F. J. Dor, L. Rydberg, and M. E. Breimer, "Studies on glycolipid antigens in small intestine and pancreas from $\alpha 1,3$-galactosyltransferase knockout miniature swine," Transplantation, vol. 84, no. 10, pp. 1348-1356, 2007.

[80] M. B. Nottle, L. F. Beebe, S. J. Harrison et al., "Production of homozygous $\alpha$-1,3-galactosyltransferase knockout pigs by breeding and somatic cell nuclear transfer," Xenotransplantation, vol. 14, no. 4, pp. 339-344, 2007.

[81] G. W. Byrne, M. G. CG, and M. E. Breimer, "Recent investigations into pig antigen and anti-pig antibody expression," International Journal of Surgery, vol. 23, Part B, pp. 223228, 2015.

[82] F. A. Neethling and D. K. C. Cooper, "Serum cytotoxicity to pig cells and anti- $\alpha \mathrm{Gal}$ antibody level and specificity in humans and baboons," Transplantation, vol. 67, no. 5, pp. 658-665, 1999.

[83] P. M. Simon, F. A. Neethling, S. Taniguchi et al., "Intravenous infusion of Gal $\alpha 1-3 \mathrm{Gal}$ oligosaccharides in baboons delays hyperacute rejection of porcine heart xenografts," Transplantation, vol. 65, no. 3, pp. 346-353, 1998.

[84] S. S. Lin, M. J. Hanaway, G. V. Gonzalez-Stawinski et al., "The role of anti-Gal $\alpha 1-3 \mathrm{Gal}$ antibodies in acute vascular rejection and accommodation of xenografts1," Transplantation, vol. 70, no. 12, pp. 1667-1674, 2000.

[85] L. E. Diamond, G. W. Byrne, A. Schwarz, T. A. Davis, D. H. Adams, and J. S. Logan, "Analysis of the control of the anti- 
Gal immune response in a non-human primate by galactose $\alpha-1-3$ galactose trisaccharide-polyethylene glycol conjugate," Transplantation, vol. 73, no. 11, pp. 1780-1787, 2002.

[86] K. Teranishi, B. Gollackner, L. Buhler et al., "Depletion of anti-Gal antibodies in baboons by intravenous therapy with bovine serum albumin conjugated to Gal oligosaccharides," Transplantation, vol. 73, no. 1, pp. 129-139, 2002.

[87] H. Xu, D. Yin, B. Naziruddin et al., "The in vitro and in vivo effects of anti-galactose antibodies on endothelial cell activation and xenograft rejection," Journal of Immunology, vol. 170, no. 3, pp. 1531-1539, 2003.

[88] N. Dujovny, A. Varghese, J. Shen et al., "Acute xenograft rejection mediated by antibodies produced independently of $\mathrm{T}_{\mathrm{H}} 1 / \mathrm{T}_{\mathrm{H}} 2$ cytokine profiles," American Journal of Transplantation, vol. 2, pp. 526-534, 2002.

[89] H. Gock, L. Murray-Segal, E. Salvaris, P. J. Cowan, and A. J. D'Apice, "Gal mismatch alone causes skin graft rejection in mice," Transplantation, vol. 74, no. 5, pp. 637-645, 2002.

[90] H. Gock, E. Salvaris, L. Murray-Segal et al., "Hyperacute rejection of vascularized heart transplants in $\mathrm{BALB} / \mathrm{c}$ Gal knockout mice," Xenotransplantation, vol. 7, pp. 237-246, 2000.

[91] H. Gock, E. Salvaris, W. Han et al., "Anti- $\alpha 1,3$-galactosemediated hyperacute rejection of vascularized transplants in a small animal model," Transplantation Proceedings, vol. 32, no. 7, p. 2075, 2000.

[92] A. M. Azimzadeh, S. S. Kelishadi, M. B. Ezzelarab et al., "Early graft failure of GalTKO pig organs in baboons is reduced by expression of a human complement pathway-regulatory protein," Xenotransplantation, vol. 22, no. 4, pp. 310-316, 2015.

[93] M. Schmoeckel, F. N. K. Bhatti, A. Zaidi et al., "Orthotopic heart transplantation in a transgenic pig-to-primate model," Transplantation, vol. 65, no. 12, pp. 1570-1577, 1998.

[94] P. D. Waterworth, J. Dunning, M. Tolan et al., "Life-supporting pig-to-baboon heart xenotransplantation," The Journal of Heart and Lung Transplantation, vol. 17, no. 12, pp. 12011207, 1998.

[95] A. Zaidi, M. Schmoeckel, F. Bhatti et al., "Life-supporting pig-to-primate renal xenotransplantation using genetically modified donors," Transplantation, vol. 65, no. 12, pp. 1584-1590, 1998.

[96] C. M. Vial, D. J. Ostlie, F. N. K. Bhatti et al., "Life supporting function for over one month of a transgenic porcine heart in a baboon," The Journal of Heart and Lung Transplantation, vol. 19, pp. 224-229, 2000.

[97] H. Xu, S. R. Gundry, W. W. Hancock et al., "Prolonged discordant xenograft survival and delayed xenograft rejection in a pig-to-baboon orthotopic cardiac xenograft model," The Journal of Thoracic and Cardiovascular Surgery, vol. 115, pp. 1342-1349, 1998.

[98] A. Bauer, H. Baschnegger, J. M. Abicht et al., "hDAF porcine cardiac xenograft maintains cardiac output after orthotopic transplantation into baboon - a perioperative study," Xenotransplantation, vol. 12, no. 6, pp. 444-449, 2005.

[99] U. Brandl, S. Michel, M. Erhardt et al., "Transgenic animals in experimental xenotransplantation models: orthotopic heart transplantation in the pig-to-baboon model," Transplantation Proceedings, vol. 39, no. 2, pp. 577-578, 2007.

[100] G. W. Byrne and C. G. McGregor, "Cardiac xenotransplantation: progress and challenges," Current Opinion in Organ Transplantation, vol. 17, no. 2, pp. 148-154, 2012.
[101] G. Byrne, "Strategy to overcome non-Gal NAb in xenotransplantation," Xenotransplantation, vol. 20, pp. 324-325, 2013.

[102] J. M. Abicht, T. Mayr, B. Reichart et al., "Pre-clinical heterotopic intrathoracic heart xenotransplantation: a possibly useful clinical technique," Xenotransplantation, vol. 22, no. 6, pp. 427-442, 2015.

[103] T. Mayr, A. Bauer, B. Reichart et al., "Hemodynamic and perioperative management in two different preclinical pig-to-baboon cardiac xenotransplantation models," Xenotransplantation, vol. 24, no. 3, 2017.

[104] A. J. Hing, A. Watson, M. Hicks et al., "Combining cariporide with glyceryl trinitrate optimizes cardiac preservation during porcine heart transplantation," American Journal of Transplantation, vol. 9, no. 9, pp. 2048-2056, 2009.

[105] S. C. Jordan and M. D. Pescovitz, "Presensitization: the problem and its management," Clinical Journal of the American Society of Nephrology, vol. 1, no. 3, pp. 421-432, 2006.

[106] C. Castleberry, T. D. Ryan, and C. Chin, "Transplantation in the highly sensitized pediatric patient," Circulation, vol. 129, no. 22, pp. 2313-2319, 2014.

[107] C. Knosalla, B. Gollackner, L. Buhler et al., "Correlation of biochemical and hematological changes with graft failure following pig heart and kidney transplantation in baboons," American Journal of Transplantation, vol. 3, no. 12, pp. 1510-1519, 2003.

[108] M. Ezzelarab, A. Cortese-Hassett, D. K. Cooper, and M. H. Yazer, "Extended coagulation profiles of healthy baboons and of baboons rejecting GT-KO pig heart grafts," Xenotransplantation, vol. 13, no. 6, pp. 522-528, 2006.

[109] D. Ricci, H. D. Tazelaar, N. Miyagi et al., "The utility of right ventricular endomyocardial biopsy for the diagnosis of xenograft rejection after CD46 pig-to-baboon cardiac transplantation," The Journal of Heart and Lung Transplantation, vol. 26, no. 10, pp. 1025-1032, 2007.

[110] A. Shimizu, Y. Hisashi, K. Kuwaki et al., "Thrombotic microangiopathy associated with humoral rejection of cardiac xenografts from $\alpha 1,3$-galactosyltransferase gene-knockout pigs in baboons," The American Journal of Pathology, vol. 172, no. 6, pp. 1471-1481, 2008.

[111] Y. Hisashi, K. Yamada, K. Kuwaki et al., "Rejection of cardiac xenografts transplanted from $\alpha 1,3$-galactosyltransferase gene-knockout (GalT-KO) pigs to baboons," American Journal of Transplantation, vol. 8, no. 12, pp. 2516-2526, 2008.

[112] L. Song, A. Ma, H. Dun et al., "Effects of ASKP1240 combined with tacrolimus or mycophenolate mofetil on renal allograft survival in cynomolgus monkeys," Transplantation, vol. 98, no. 3, pp. 267-276, 2014.

[113] G. R. Gunn 3rd, D. C. Sealey, F. Jamali, B. Meibohm, S. Ghosh, and G. Shankar, "From the bench to clinical practice: understanding the challenges and uncertainties in immunogenicity testing for biopharmaceuticals," Clinical and Experimental Immunology, vol. 184, no. 2, pp. 137-146, 2016.

[114] M. A. Evans, P. J. Schomberg, R. J. Rodeheffer et al., "Total lymphoid irradiation: a novel and successful therapy for resistant cardiac allograft rejection," Mayo Clinic Proceedings, vol. 67, pp. 785-790, 1992.

[115] D. H. Sachs, R. B. Colvin, A. B. Cosimi et al., "Xenotransplantation-caution, but no moratorium," Nature Medicine, vol. 4, no. 4, pp. 372-373, 1998. 
[116] C. Patience, Y. Takeuchi, and R. A. Weiss, "Infection of human cells by an endogenous retrovirus of pigs," Nature Medicine, vol. 3, no. 3, pp. 282-286, 1997.

[117] O. Stephan, J. Schwendemann, V. Specke, S. J. Tacke, K. Boller, and J. Denner, "Porcine endogenous retroviruses (PERVs): generation of specific antibodies, development of an immunoperoxidase assay (IPA) and inhibition by AZT," Xenotransplantation, vol. 8, pp. 310-316, 2001.

[118] M. Niebert, C. Rogel-Gaillard, P. Chardon, and R. R. Tonjes, "Characterization of chromosomally assigned replicationcompetent gamma porcine endogenous retroviruses derived from a large white pig and expression in human cells," Journal of Virology, vol. 76, no. 6, pp. 2714-2720, 2002.

[119] M. A. Groenen, A. L. Archibald, H. Uenishi et al., "Analyses of pig genomes provide insight into porcine demography and evolution," Nature, vol. 491, no. 7424, pp. 393-398, 2012.

[120] B. Bartosch, R. A. Weiss, and Y. Takeuchi, "PCR-based cloning and immunocytological titration of infectious porcine endogenous retrovirus subgroup $\mathrm{A}$ and $\mathrm{B}$," The Journal of General Virology, vol. 83, pp. 2231-2240, 2002.

[121] C. Patience, G. S. Patton, Y. Takeuchi et al., "No evidence of pig DNA or retroviral infection in patients with short-term extracorporeal connection to pig kidneys," Lancet, vol. 352, no. 9129, pp. 699-701, 1998.

[122] Z. Pitkin and C. Mullon, "Evidence of absence of porcine endogenous retrovirus (PERV) infection in patients treated with a bioartificial liver support system," Artificial Organs, vol. 23, no. 9, pp. 829-833, 1999.

[123] J. H. Dinsmore, C. Manhart, R. Raineri, D. B. Jacoby, and A. Moore, "No evidence for infection of human cells with porcine endogenous retrovirus (PERV) after exposure to porcine fetal neuronal cells," Transplantation, vol. 70, no. 9, pp. 1382-1389, 2000.

[124] L. Scobie, V. Padler-Karavani, S. Le Bas-Bernardet et al., "Long-term IgG response to porcine Neu5Gc antigens without transmission of PERV in burn patients treated with porcine skin xenografts," Journal of Immunology, vol. 191, no. 6, pp. 2907-2915, 2013.

[125] U. Martin, S. J. Tacke, A. R. Simon et al., "Absence of PERV specific humoral immune response in baboons after transplantation of porcine cells or organs," Transplant International, vol. 15, no. 7, pp. 361-368, 2002.

[126] J. Denner and N. J. Mueller, "Preventing transfer of infectious agents," International Journal of Surgery, vol. 23, Part B, pp. 306-311, 2015.

[127] A. G. Katopodis, R. G. Warner, R. O. Duthaler et al., "Removal of anti-Gal $\alpha 1,3 \mathrm{Gal}$ xenoantibodies with an injectable polymer," The Journal of Clinical Investigation, vol. 110, pp. 1869-1877, 2002.

[128] G. W. Byrne, A. Schwarz, J. R. Fesi et al., "Evaluation of different $\alpha$-galactosyl glycoconjugates for use in xenotransplantation," Bioconjugate Chemistry, vol. 13, no. 3, pp. 571581, 2002.

[129] E. Cozzi and D. J. G. White, "The generation of transgenic pigs as potential organ donors for humans," Nature Medicine, vol. 1, no. 9, pp. 964-966, 1995.
[130] L. E. Diamond, C. M. Quinn, M. J. Martin, J. H. Lawson, J. L. Platt, and J. S. Logan, "A human CD46 transgenic pig model system for the study of discordant xenotransplantation," Transplantation, vol. 71, no. 1, pp. 132-142, 2001.

[131] B. E. Loveland, J. Milland, P. Kyriakou et al., "Characterization of a CD46 transgenic pig and protection of transgenic kidneys against hyperacute rejection in non-immunosuppressed baboons," Xenotransplantation, vol. 11, no. 2, pp. 171-183, 2004. 


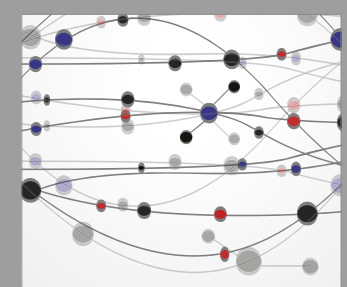

The Scientific World Journal
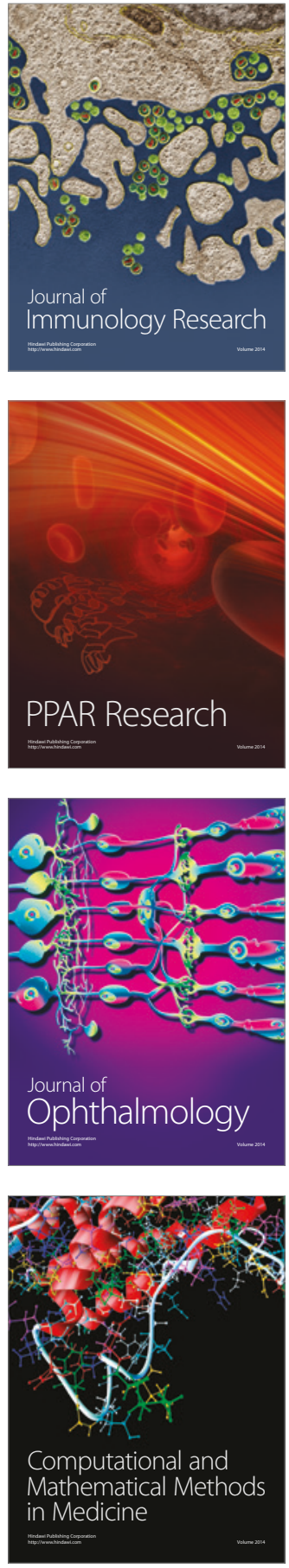

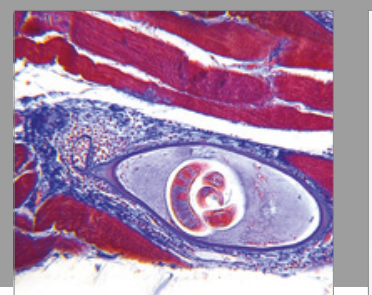

Gastroenterology Research and Practice
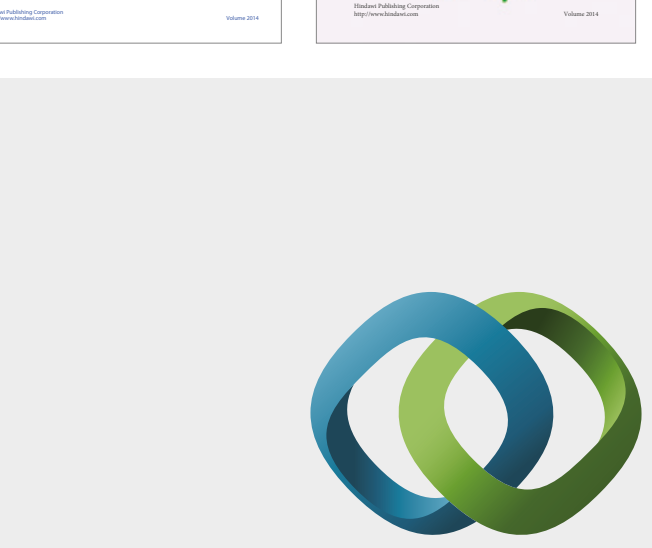

\section{Hindawi}

Submit your manuscripts at

https://www.hindawi.com
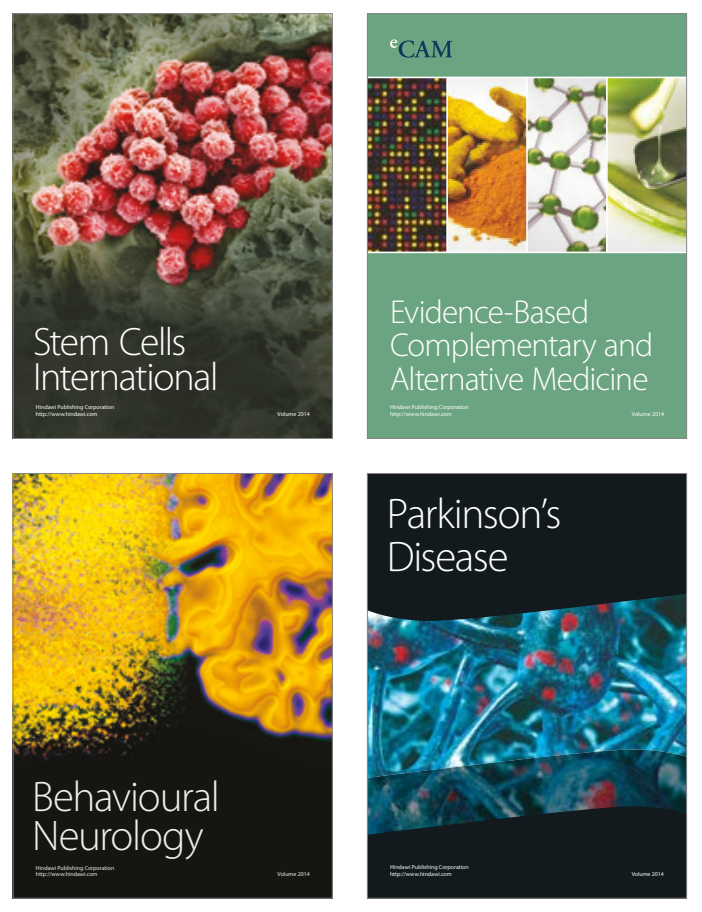
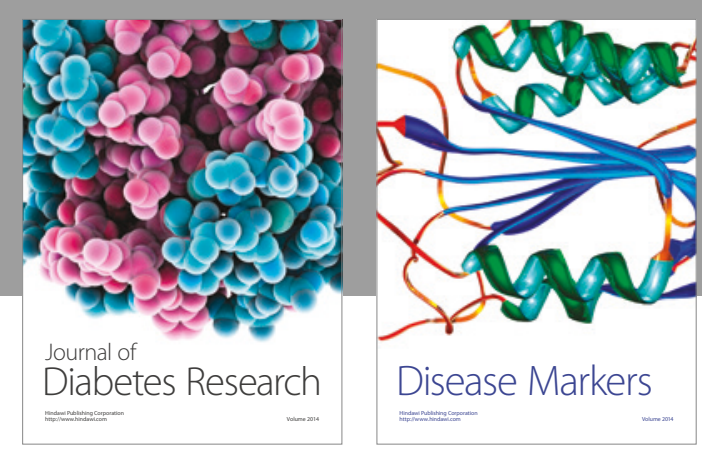

Disease Markers
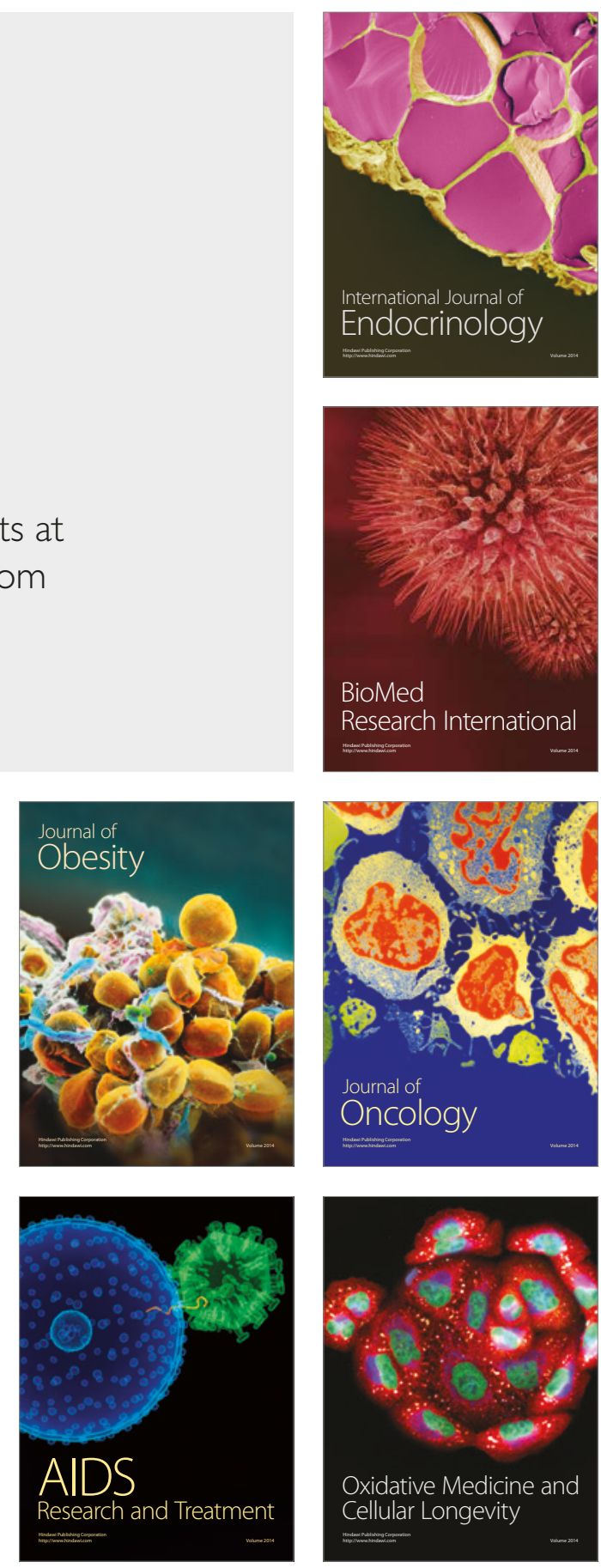